\title{
Conocimientos, prácticas y actitudes de enfermería para la atención de personas con tuberculosis
}

\section{Nursing knowledge, practices, and attitudes regarding the attention of persons with tuberculosis}

\section{Conhecimentos, práticas e atitudes de enfermagem para a atenção de pessoas com tuberculose}

F. Rodríguez-Mora ${ }^{a *}$, S. Sánchez-Piña ${ }^{\text {b2 }}$

ORCID

${ }^{a}$ https://orcid.org/0000-0002-5018-3859

${ }^{b}$ https://orcid.org/0000-0002-5659-8940

\begin{abstract}
${ }^{1}$ Universidad Nacional Autónoma de México, Escuela Nacional de Enfermería y Obstetricia, Programa de Maestría en Enfermería, Ciudad de México, México

2Universidad Nacional Autónoma de México, Escuela Nacional de Enfermería y Obstetricia, Sistema de Universidad Abierta y Educación a Distancia, Ciudad de México, México
\end{abstract}

Recibido: 25 julio 2019 / Aceptado: 3 diciembre 2019

\section{RESUMEN}

Introducción: La tuberculosis es un padecimiento con una gran carga económica y social; representa una de las 10 causas principales de mortalidad a nivel mundial. Múltiples factores intervienen en la adherencia al tratamiento y cura de la enfermedad. La atención de enfermería estudiada desde 3 indicadores (conocimiento, práctica y actitud), que son determinantes cruciales para el cumplimiento de una atención de calidad y para la implementación de nuevas tecnologías de cuidados; es imprescindible.

Objetivo: Evaluar los conocimientos, prácticas y actitudes de enfermería relacionados con la atención de pacientes con tuberculosis.

Metodología: Estudio cuantitativo, descriptivo, muestra no probabilística de 19 enfermeras(os) del primer nivel de atención. Se evaluaron los conocimientos básicos generales, los relacionados con el diagnóstico y con el tratamiento. Las prácticas comprendieron la identificación de recursos para el registro, las visitas, la elaboración de planes de cuidados 
de enfermería, además del uso de una herramienta digital que se brindó para facilitar la elaboración de dichos planes. En actitudes, se evaluaron tres componentes: cognitivo, conductual y afectivo.

Resultados: Se identificó una base débil de conocimientos en tuberculosis; la mayor parte mostró un nivel de conocimiento medio y bajo. Poco más de la mitad de los participantes se ubicó con una práctica regular y, de manera general, la actitud al brindar los cuidados fue buena. Conclusiones: Se requiere fortalecer la atención de enfermería en tuberculosis desde los tres indicadores evaluados. Sin una base consolidada de conocimientos sobre la enfermedad es difícil alcanzar los objetivos de los programas y las políticas en salud pública; esto, a su vez, repercute de manera directa en la práctica y actitud de los profesionales.

Palabras clave: Tuberculosis; atención de enfermería; plan de cuidados estandarizado; México.

ABSTRACT

Introduction: Being one of the 10 main causes of mortality worldwide, tuberculosis causes a great economic and social burden to societies. Considering that diverse factors are involved in the treatment adherence, and consequent cure, nursing attention, assessed through the related knowledge, practices, and attitudes, becomes of paramount importance.

Objective: To assess the tuberculosis-related knowledge, practices, and attitudes among nurses.

Methodology: This is a quantitative and descriptive study with a non-probabilistic sample of 19 nurses in primary care. Regarding knowledge, the basic general, and diagnosis and treatment related were assessed. Regarding practices, the resources identification, visits, care plans, and digital tools use were assessed. Regarding attitudes, the cognitive, behavioral, and affective areas were assessed.

Results: The majority of nurses demonstrated a fair or low level of tuberculosis related knowledge. A little more than half of the participants demonstrated a regular or fair level of tuberculosis related practice. In general, the attitudes towards providing tuberculosis care were good.

Conclusions: It is necessary to strengthen the tuberculosis related nursing attention in terms of the 3 indicators assessed in this study. Without a sound base of knowledge, an expertised practice, and a good attitude related to addressing the problem of tuberculosis, it is hard to accomplish the objectives, programs, and policies of Public Health, a situation which indirectly, can also have negative impacts on the health professionals.

Keywords: Tuberculosis; nursing care; standardized care plans; Mexico.

\section{RESUMO}

Introdução: A tuberculose é uma doença com uma grande carga económica e social, representa uma das 10 causas principais de mortalidade a nível mundial. Múltiplos fatores intervêm na aderência ao tratamento e cura da doença. A atenção de enfermagem estudada desde 3 indicadores (conhecimento, prática e atitude), que são determinantes cruciais para o cumprimento de uma atenção de qualidade e para a implementação de novas tecnologias de cuidados, é imprescindível.

Objetivo: Avaliar os conhecimentos, práticas e atitudes de enfermagem relacionados com a atenção de pacientes com tuberculose. 
Metodologia: Estudo quantitativo, descritivo, amostra não probabilística de 19 enfermeiras (os) do primeiro nível de atenção. Avaliaram-se os conhecimentos básicos gerais, os relacionados com o diagnóstico e, com o tratamento. As práticas compreenderam a identificação de recursos para o registro, as visitas, a elaboração de planos de cuidados de enfermagem, além disso do uso de uma ferramenta digital que se proporcionou para facilitar a elaboração desses planos. Em atitudes avaliaram-se três componentes: cognitivo, comportamental e afetivo. Resultados: Identificou-se uma base de conhecimentos em tuberculose fraca, a maior parte mostrou um nível de conhecimento médio e baixo. Pouco mais da metade dos participantes situou-se com uma prática regular e de maneira geral, a atitude de proporcionar os cuidados foi adequada.

Conclusões: É preciso fortalecer a atenção de enfermagem em tuberculose desde os três indicadores avaliados. Sem uma base consolidada de conhecimentos sobre a doença é difícil atingir os objetivos dos programas e as políticas em saúde pública; isto por sua vez, repercute de forma direta na prática e atitude dos professionais.

Palavras chave: Tuberculose; cuidados de enfermagem; plano de cuidados estandardizado; México.

\section{INTRODUCCIÓN}

La tuberculosis (TB) es un padecimiento con una gran carga económica ${ }^{1-2}$ y social ${ }^{3}$ a nivel internacional; de acuerdo con el Global Tuberculosis Report, en 2017 se estimaron 10 millones de casos nuevos en el mundo (5.8 millones hombres, 3.2 millones mujeres y 1.0 millones en niños), además de registrarse 1.3 millones de muertes a causa de la enfermedad4. Según la última estadística del Centro Nacional de Programas Preventivos y Control de Enfermedades (CENAPRECE) de México, en 2016 se documentaron 16 mil 913 casos nuevos de TB pulmonar, 373 TB meníngea y 3 mil 898 TB en otras formas 5 .

La TB representa una de las 10 causas principales de mortalidad a nivel mundial ${ }^{6}$; si bien se conoce que el agente causal es la micobacteria Mycobacterium tuberculosis, varios expertos han encontrado que diversos patrones epidemiológicos propician la propagación de la enfermedad y dificultan la adherencia al tratamiento; por ejemplo: el flujo de inmigración puede aumentar los casos de TB en países industrializados; la resistencia a los fármacos como la TB multi-drogorresistente (TB-MDR), ésta con altos índices en Europa oriental, y el binomio TB-VIH presente en África y Asia7.

La TB es una enfermedad contagiosa, crónica y de transmisión rápida; el medio de contagio es por gotas de saliva que provienen de tos o un estornudo de una persona enferma. En México, en 2012 la incidencia fue de 16.8 por cada 100 mil habitantes, afecta principalmente el tejido pulmonar y la población más sensible a padecerla se encuentra entre 15-49 años. En cuanto a la mortalidad, se registraron 253 muertes con una tasa de 1.9 por cada 100 mil habitantes ${ }^{8}$.

Debido a lo anterior y al acelerado crecimiento de la población alrededor del mundo, así como la reemergencia y aparición de nuevas enfermedades, se ha producido un incremento en la demanda de atención especializada y eficaz de enfermería, con el propósito de buscar alternativas innovadoras que permita a dicho personal coadyuvar en la recuperación de estos pacientes.

Entre los diversos factores asociados a la problemática de la práctica de enfermería en la atención de pacientes con TB, se han reportado ciertos elementos que dificultan el cumplimiento de brindar atención de calidad; entre ellos se encuentran: déficit de conocimientos en $\mathrm{TB}^{9,10}$, prácticas de riesgo durante la atención de estos pacientes ${ }^{11,12}$, un marcado estigma social ante la enfermedad 
y las personas que la padecen. Lo anterior repercute en la actitud de los profesionales ${ }^{12} \mathrm{y}$ ésta en el apego al tratamiento y cura de la enfermedad.

La dificultad y la falta de tiempo para emplear el Proceso de Atención de Enfermería (PAE) evitan un seguimiento idóneo y continuo de los cuidados en la práctica ${ }^{13-15}$. Es por ello que, en $2008^{16}$ en México, el Sistema de Calidad Integral en Salud (SICALIDAD) incluye dentro del eje de Calidad técnica y seguridad del paciente, una línea de acción denominada Plan de Cuidados de Enfermería (PLACE); su importancia para la profesión reside en el valor científico y legal que se le atribuye para obtener evidencia e información documentada de los cuidados realizados; aunque no por ello es aplicado exitosamente por los profesionales en enfermería.

Vinculada a esta necesidad, la implementación de herramientas estructuradas específicas para atención de la TB permitirían identificar las necesidades de los pacientes y que éstas puedan ser atendidas a la brevedad; sobre todo porque es un padecimiento, el cual, si no es tratado de forma inmediata, puede transmitirse rápidamente; por tal motivo se ha considerado preciso ampliar los diseños metodológicos de los instrumentos de valoración, con el propósito de mejorar la calidad de la atención de los pacientes con TB.

Debido a lo anterior, un grupo de enfermeras de la Escuela Nacional de Enfermería y Obstetricia, UNAM (ENEO-UNAM), en conjunto con ingenieros en informática, desarrollaron una aplicación digital que facilitara la realización de Planes de Cuidados de Enfermería estandarizados que guíen la atención de personas con TB; esta planeación toma como fundamento el Modelo Conceptual de Virginia Henderson y tiene como antecedente la validación de un instrumento para valorar a personas con $\mathrm{TB}^{17}$.

El Sistema Experto en Tuberculosis (SE), nombre de la aplicación, se diseñó para contener los rubros correspondientes a las 14 Necesidades de Henderson con las manifestaciones a valorarse en cada una de éstas; tiene además integrado un subconjunto de las taxonomías NANDA, NOC, NIC (67 etiquetas diagnósticas, 59 diagnósticos y de 8 a 13 intervenciones por cada diagnóstico), los cuales conforman la base de conocimientos del $\mathrm{SE}^{17}$. Puede ser utilizado en computadoras, tabletas y teléfonos móviles con sistema operativo Android versión mínima 4. $42^{18}$.

El uso de una aplicación como ésta, se encuentra en concordancia con los planteamientos que la OMS ${ }^{1}$ indica a través del informe mundial en TB, pues la innovación es fundamental para el futuro de la respuesta a esta gran necesidad social. El cambio del modelo cultural por el que atraviesa la sociedad en la actualidad ha llevado a que se presenten diversas modificaciones en cuanto a la manera de comunicarse, aprender, enseñar, así como de cuidarse. Por ello, este organismo mundial reconoce que todos los días la salud humana mejora gracias a las TIC ${ }^{22}$.

Se caracterizó la atención de acuerdo con los programas de salud pública estandarizados, mediante tres indicadores:

- El conocimiento en TB,

- Las prácticas, científicamente fundamentadas para los cuidados autónomos y en colaboración interdependiente que se brinda a la persona, familia y comunidad, enfermos o sanos, en todos los contextos ${ }^{19}$; se basa en la priorización de las necesidades y de forma que promueva una plena participación de los mismos en su recuperación y,

- La actitud del profesional ante la atención a personas con TB.

De acuerdo con lo explicado previamente, esta investigación tiene como objetivo evaluar conocimientos, prácticas y actitudes de enfermería vinculadas a la atención de personas con tuberculosis. 


\section{METODOLOGÍA}

Estudio cuantitativo, descriptivo ${ }^{23}$. Con una muestra de 19 enfermeras (os), los criterios de inclusión fueron: personal de enfermería activo en centros de salud del primer nivel de atención, que tuvieran pacientes con TB y que estuvieran dispuestos a participar voluntariamente. Exclusión: enfermeras (os) que no desearan participar en la investigación, que no contaran con un teléfono celular con los requerimientos para el uso del SE. Previo a la selección de participantes, se había indagado si utilizaban la computadora, el teléfono celular y la tableta para actividades de la vida diaria; se encontró que el 90\% no utiliza la tableta, 100\% maneja el teléfono celular y 90\% la computadora. Al indagar si habían utilizado alguno de estos tres dispositivos para funciones específicas de la práctica de enfermería, solamente $37 \%$ los usó y $63 \%$, no.

Se utilizó un instrumento para la recolección de datos creado por las autoras, Atención de enfermería a pacientes con tuberculosis con el que se realizó una prueba piloto y se obtuvo una confiabilidad de Alpha de Cronbach de 0.89 .

El instrumento está conformado por una sección de datos generales (sexo, jurisdicción a la que pertenece el lugar de trabajo, antigüedad en el trabajo, formación académica). Por otra parte, evalúa conocimiento en TB mediante 15 reactivos (1-5 asociados a preguntas acerca de la enfermedad, 6-10 referente a los medios de diagnóstico y 11-15 concerniente al tratamiento antituberculosis) en escala dicotómica. Los resultados se clasificaron en: bajo < 8 puntos, medio 9-11 puntos y alto 12-15 puntos.

Prácticas de enfermería. Entre éstas, se incluyó un ítem para identificar el uso o no de una aplicación (Sistema Experto) que fue proporcionada al personal de enfermería que aceptó participar en el estudio. Cabe señalar que la evaluación específica de la facilidad, percepción de su utilidad, así como tiempo invertido en la elaboración del PLACE con la aplicación fueron motivo de otro estudio. Así, las prácticas de enfermería se evaluaron a partir de siete reactivos: 1) disponibilidad de formatos de valoración; 2) documentación de los cuidados; 3) dotación de insumos para la visita domiciliaria; 4) recursos para trasladarse a las viviendas; 5) distribución del recurso humano para la atención de personas con TB; 6) frecuencia de aplicación del PLACE a personas con TB antes de contar con la aplicación; 7) frecuencia de uso del PLACE posterior a tener la aplicación. Las opciones de respuesta se formularon con una escala tipo likert (nunca o, casi nunca 1, algunas veces 2, casi siempre 3, siempre 4); para el caso de la pregunta de distribución del personal la escala de medición fue (mala o, deficiente 1, regular 2, buena 3, excelente 4), un total de 28 puntos en esta sección.

Por último, la actitud del profesional durante la atención fue evaluada en escala tipo Likert (nunca 0 , casi nunca 1, algunas veces 2, casi siempre 3, siempre 4) mediante 17 reactivos (1-7 componente cognitivo, 8-12 componente conductual y 13-17 componente afectivo) para un total de 68 puntos. La escala de clasificación de los resultados (nivel de actitud) fue: mala < 44; regular 45-55; buena, 56-68 puntos.

Para el procesamiento de la información se utilizó el programa SPSS V.24, estadística descriptiva e inferencial (pruebas no paramétricas). La presente investigación se realizó bajo los mandatos del Reglamento de la Ley General de Salud en Materia de Investigación para la Salud ${ }^{24}$; fue clasificada como investigación sin riesgo para la salud e integridad de las personas participantes, se contempló el respeto al consentimiento informado de manera verbal y escrita, así como el derecho a retirarse del estudio. El proyecto fue presentado ante la Dirección de Investigación y Educación de la Secretaría de Salud de la Ciudad de México, y fue aprobado por el Comité de Ética registrado con el número 102-110-02-17. 


\section{RESULTADOS}

La muestra estuvo conformada por 19 participantes con una edad media de 37.2, ( \pm 7.6$)$, mínimo 23 y máximo 57 años; solamente un 5\% corresponde al sexo masculino. El nivel de formación predominante es de licenciatura; poco más de la mitad había recibido capacitación previa respecto al manejo de pacientes con tuberculosis; dos terceras partes tienen 6 o más años de antigüedad laboral en la Unidad (Tabla 1).

Tabla 1. Características sociodemográficas

\begin{tabular}{lrr}
\hline Variable & Fo & $\%$ \\
\hline Sexo & & \\
\hline Femenino & 18 & 95 \\
Masculino & 1 & 5 \\
\hline Grupos de edad & & 47 \\
\hline 20 a 35 & 9 & 47 \\
36 a 49 & 9 & 6 \\
50 o más & 1 & 21 \\
Formación académica & & 63 \\
\hline Carrera Técnica & 4 & 11 \\
Lic. en Enfermería & 12 & 5 \\
Especialidad & 2 & \\
Maestría en Enfermería & 1 & 48 \\
\hline Capacitaciones previas en TB & & \\
\hline Sí & 11 & 32 \\
No & 8 & 42 \\
\hline Antigüedad Laboral en la Unidad de Salud (años) & & 26 \\
\hline 1 a 5 & 6 & \\
6 a 10 & 8 & \\
11 o más & 5 & \\
\hline
\end{tabular}

A continuación, se desglosan los tres indicadores que para este estudio se vinculan con la atención de enfermería en TB:

\section{Conocimiento en TB}

Se evaluaron tres principales temáticas: conocimientos básicos de la enfermedad, de los métodos de diagnóstico y del esquema farmacológico.

Respecto al nivel de conocimiento de los participantes, se detectó una base débil de conocimientos en TB, ya que 43\% quedó clasificado en el nivel bajo, 47\% en el nivel medio y únicamente un 10\% se situó en un nivel alto.

De los participantes, $68 \%$ no identificó correctamente qué comprende el esquema farmacológico del tratamiento antituberculosis; un 37\% ignora las reacciones adversas a los medicamentos de primera línea, otro $37 \%$ desconoce la causa a la resistencia de los fármacos antituberculosis. Por otro lado, el 90\% identificó correctamente los medicamentos de primera línea.

En cuanto a los métodos de diagnóstico, $58 \%$ desconoce las condiciones en las que se deben mantener las muestras de expectoración; 32\% no identifica la técnica diagnosticar la TB renal y; 74\% no domina las acciones a realizar cuando en el control baciloscópico de un paciente bajo tratamiento se encuentra el siguiente esquema: 1er mes positivo, $2^{\circ}$ mes negativo, $3 \mathrm{er}$ mes negativo y $4^{\circ}$ mes positivo (presencia de 1 a 2 bacilos en la muestra). A su vez, todos identifica correctamente el método diagnóstico para la TB pulmonar y 90\% conoce con qué frecuencia deben realizar los estudios de expectoración en caso de TB pulmonar. 
Por último, 53\% desconoce cuánto tiempo después se realiza la lectura a la reacción del PPD (Derivado proteico purificado); 96\% identificó correctamente el agente etiológico de la TB; 84\% reconoce los síntomas frecuentes de la TB pulmonar y los mecanismos de trasmisión; el 96\% conoce los intervalos de administración del tratamiento entre cada dosis hasta completar 45 en la fase de sostén del Tratamiento Acortado Estrictamente Supervisado (TAES).

No se encontraron diferencias estadísticas en el nivel de conocimiento según la edad $\mathrm{X}^{2}=1.872$, $\mathrm{gl}=2, \mathrm{p}=0.392$; antigüedad laboral $\mathrm{X}^{2}=0.582, \mathrm{gl}=2, \mathrm{p}=0.748$; o si habían recibido capacitaciones previas en TB $Z=-0.791, p=0.429$.

\section{Práctica de enfermería en TB}

El 58\% del personal manifestó una práctica regular y el 42\% en buena. Se evaluaron los siguientes aspectos:

1. Formatos de valoración: $15 \%$ dijo nunca o casi nunca contar con ellos; $10 \%$ algunas veces y $75 \%$ casi siempre o siempre cuentan con formatos.

2. Documentación de los cuidados: $6 \%$ casi nunca documenta los cuidados realizados; $21 \%$ algunas veces y $73 \%$ casi siempre o siempre.

3. Recursos para la visita domiciliaria: a) Dotación de insumos: $5 \%$ casi nunca; $11 \%$ algunas veces y $84 \%$ casi siempre o siempre cuentan con los insumos necesarios; b) Recursos para trasladarse a las viviendas: $26 \%$ nunca o casi nunca; $16 \%$ algunas veces y $58 \%$ casi siempre o siempre.

5. Distribución del recurso humano en TB: $47 \%$ refirió ser buena; $37 \%$ regular; $10 \%$ deficiente y $6 \%$ mala.

6, 7. Frecuencia de aplicación del PLACE en pacientes con TB: Se indagó la frecuencia de su utilización antes de contar con la aplicación digital y posterior a tenerla; $64 \%$ dijo casi siempre o siempre aplicar el PLACE en TB (por medios tradicionales) mientras que, posterior al uso del SE el 95\%, dijo casi siempre o siempre aplicar el PLACE en TB (con el SE). Simultáneamente, 20\% dijo nunca o casi nunca aplicar el PLACE en TB antes de tener el SE, mientras que, al contar con él, la categoría de nunca o casi nunca fue o\%. (Gráfica 1).

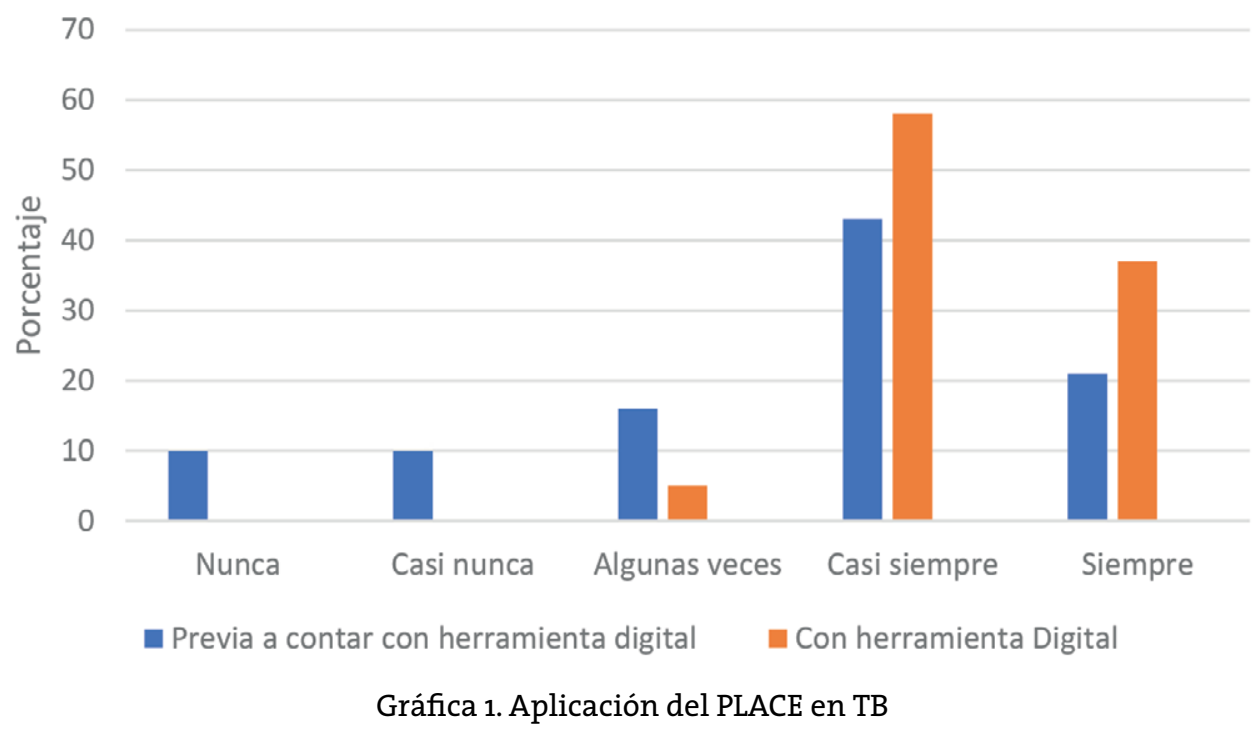


Se encontraron diferencias estadísticas en la aplicación del PLACE en TB posterior al uso del Sistema Experto; al realizar la prueba de Wilcoxon se obtuvo $Z=-2.208, p=0.027$.

\section{Actitud del profesional durante la atención}

En cuanto a la actitud durante la atención, se estudió desde 3 componentes (cognitivo, conductual y afectivo). El personal de enfermería presentó una actitud favorable: $63 \%$ se clasificó como buena; $32 \%$, regular y $5 \%$, mala.

\section{DISCUSIÓN}

El conocimiento es fundamental para el cumplimiento y desarrollo de cualquier tarea o práctica de calidad según el área de desempeño; el déficit de conocimientos básicos en TB es un problema que trasciende a nivel internacional, incluso a otras disciplinas del ámbito de la salud. En esta investigación, se detectó una base débil para el cuidado de la persona con TB; el nivel de conocimiento de enfermería se encontró entre medio y bajo principalmente, lo que puede repercutir en la eficiencia y eficacia de los cuidados que se brindan. Lo anterior coincide con lo reportado por Cruz Martínez et $a .{ }^{25}$ en Colombia, quienes evaluaron el conocimiento en tuberculosis de los trabajadores de la salud (enfermeras, auxiliares de enfermería, médicos, técnicos de laboratorio, etc.) que laboraban en hospitales de segundo nivel y centros de atención primaria. El 59\% de su muestra obtuvo un nivel de conocimiento bajo; $34 \%$, medio y $7 \%$, alto.

Una base de conocimientos fundamentada, actualizada y correcta es esencial para asegurar el éxito del tratamiento y cura de la enfermedad; de lo contrario, se convierte en una situación perjudicial, ya que los esquemas farmacológicos inadecuados a menudo son la principal causa de la TB MDR, misma que genera elevados costos para el sistema de salud y los pacientes.

Como parte de los procesos de mejora en pro del cumplimiento de los objetivos del desarrollo sostenible en TB, el conocimiento actualizado, preciso, correcto de los profesionales de enfermería garantiza una oportuna orientación y acompañamiento a las personas con esta enfermedad, a sus familias y a las comunidades, lo que permite asegurar el éxito del tratamiento antituberculosis ${ }^{26}$.

En la dimensión de conocimientos, en el ítem esquema farmacológico, 68\% de los participantes no logró identificar qué comprende el esquema de la Red TAES, lo cual puede incrementar la posibilidad en los pacientes de desarrollar MDR. En estudios previos ${ }^{11,27}$, se han encontrado resultados similares de un nivel de conocimiento bajo en relación con la administración del tratamiento antituberculosis por los trabajadores de la salud.

Los registros de enfermería constituyen un documento legal y son la evidencia escrita de aquellas intervenciones llevadas a cabo en los pacientes, por lo que la calidad de éstos pone de manifiesto una comunicación efectiva para propiciar la continuidad y coordinación efectiva entre los miembros del equipo de salud ${ }^{28}$.

De igual forma, se considera que el registro de las intervenciones de enfermería es trascendental para la continuidad en la atención. Sin embargo, únicamente el 26\% en este estudio refirió documentar siempre las intervenciones; situación que se asemeja a lo encontrado por Torres Santiago en $2011^{28}$, quien estudió los registros de enfermería y obtuvo un índice de eficiencia global de $72 \%$, el cual, de acuerdo con los estándares, corresponde a un nivel de cumplimiento mínimo de registro, lo que alerta de la necesidad de concientización del personal para un registro de calidad, que le permita a los diferentes miembros del equipo de salud involucrados en el cuidado, llevar un seguimiento oportuno y una adecuada toma de decisiones. 
El uso de una herramienta adicional, que en este caso corresponde a la aplicación digital implementada, puede interpretarse como una contribución que va en el sentido de establecer estándares e indicadores de calidad al tener como base la ciencia de la implementación, donde enfermeras (os) gestores del primer nivel de atención puedan planificar, analizar, diseñar e intervenir sobre aquellos procesos y factores que se asocian a una integración efectiva de intervenciones basadas en evidencia, en un contexto clínico o comunitario en particular ${ }^{29}$; así como utilizar herramientas que permitan la identificación de riesgos y actuar de manera proactiva y reactiva.

La literatura reporta diferentes experiencias y hallazgos en cuanto a la actitud de los trabajadores de la salud en la atención de TB; por un lado, en Irán ${ }^{11}$ obtuvieron una buena actitud en general asociada a la formación académica, mientras que Carvajal Barona et al., en Colombia ${ }^{12}$, encontraron que la actitud fue negativa hacia la enfermedad. Existe un fuerte sentimiento de estigma en los trabajadores y otra positiva, asociada a la educación de pacientes y familiares.

En este estudio se encontró que $63 \%$ de los participantes tenía una buena actitud en la atención, lo cual puede coincidir con los resultados de Droosti ${ }^{11}$; sin embargo, el $37 \%$ que se encuentra con una actitud mala o regular, coincide con los hallazgos de Carvajal Barona ${ }^{12}$.

\section{CONCLUSIONES}

El estudio de la evidencia basado en la práctica, así como las posibles limitaciones y fortalezas constituye el camino de las nuevas investigaciones en la disciplina de enfermería y en el ámbito de salud pública. Se requiere establecer estrategias, políticas y planes de trabajo que permitan dirigir la práctica profesional del primer nivel de atención hacia el logro de los objetivos, y situarse como una disciplina competitiva y de práctica avanzada.

Se cumplió con el objetivo planteado al evaluar conocimientos, prácticas y actitudes de enfermería vinculadas a la atención de personas con tuberculosis, con lo que se pueden establecer procesos para mejorar la calidad de la atención.

El éxito del tratamiento antituberculosis depende en gran medida de la adherencia por parte de los pacientes y de la correcta administración, además de la supervisión de enfermería; por tanto, el conocimiento se torna un factor crucial para el cumplimiento de una atención segura y de calidad.

Sin embargo, estos resultados deben interpretarse con mesura debido a algunas limitaciones metodológicas en la selección de la muestra, por lo que no se pueden generalizar a todas las enfermeras (os) del primer nivel de atención.

Se comprobó que el Sistema Experto fue un factor que permitió incrementar la aplicación del PLACE en pacientes con TB. Es una herramienta que puede coadyuvar en la prevención de posibles riesgos en la curación de los pacientes, una continuidad de los cuidados y evaluación del progreso de éstos, además de registrar y hacer visible el trabajo del capital humano de enfermería.

La práctica de enfermería requiere de herramientas que apoyen los cuidados que la tornen en eficiente, continua y comprensible para todos los involucrados. Que sea un medio de comunicación y facilite la toma de decisiones para futuras valoraciones y planes de cuidados.

La actitud del profesional durante la atención tiene una repercusión en la apreciación de la calidad de los servicios. De ahí la importancia de identificar los factores que influencian el uso efectivo y completo de las innovaciones científicas en la práctica, las cuales intentan maximizar los beneficios de las intervenciones y programas de políticas públicas en salud. 
Aunque el SE fue una herramienta de apoyo para el plan de cuidados, pudiera generar cambios en cada indicador evaluado y un aumento en el conocimiento básico de la enfermedad; en las prácticas, obtener un mayor registro del PLACE en TB, impactaría de manera favorable en la actitud del profesional. Evaluar esto, da pie a nuevas investigaciones.

En términos generales, podríamos decir que la atención de enfermería considerada como la sumatoria de los tres indicadores (conocimiento, prácticas y actitud) en el paciente con TB, requiere que las futuras investigaciones sean en torno a la evidencia basada en la práctica y que sirva de punto de referencia para la toma de decisiones, nuevas políticas y programas de acción.

\section{RESPONSABILIDADES ÉTICAS}

Protección de personas y animales. Las autoras declaran que en este estudio no se realizaron experimentos en seres humanos ni animales.

Confidencialidad. En este estudio no aparecen datos que permitan identificar a los participantes.

Derecho a la privacidad y consentimiento informado. Las autoras declaran que han obtenido el consentimiento informado de los participantes del estudio.

Conflicto de intereses. Las autoras declaran no tener conflicto de intereses.

Financiamiento. A través de un proyecto PAPIIT IT 202614.

Agradecimientos. A la Coordinación Normativa de Enfermería de los Servicios de Salud Pública de la Ciudad de México por su apoyo incondicional y al grupo de enfermeras que participaron en esta investigación.

\section{REFERENCIAS}

1. OMS. Informe Mundial sobre la Tuberculosis 2017. Ginebra: OMS; 2017. https://bit.ly/2BDBoGV

2. Unión Internacional contra la Tuberculosis y Enfermedades Respiratorias. Respuesta de la Unión al Informe mundial sobre tuberculosis 2018 de la OMS. https://bit.ly/2DoUN5d

3. León-Cabrera P, Pría-Barros MC, Perdomo-Victoria I, Ramis-Andalia R. Aproximación teórica a las desigualdades sociales en la tuberculosis como problema de salud. Rev Cubana Salud Pública. 2015; 41(3): 532-46. http://bit.ly/2wgitmH

4. WHO. Global Tuberculosis Report 2018. Geneva: WHO; 2018. https://bit.ly/2NRCjXE

5. Secretaría de Salud. Centro Nacional de Programas Preventivos y Control de Enfermedades. Cifras oficiales tuberculosis. México: Secretaría de Salud; 2017. http://bit.ly/2qLsyTQ

6. OMS. Tuberculosis. Datos y cifras 2019. Ginebra: OMS; 2019. https://bit.ly/350uHoF

7. Morales N, Beldarraín Chaple E. Aspectos económicos y sociales relacionados con el comportamiento de la tuberculosis en Cuba. Década de los 9o. Medisur. 2015; 13(2): 239-47. https://bit.ly/2OxiODxF

8. Secretaría de Salud. Programa de Acción Específico. Prevención y Control de la Tuberculosis 20132018. México: Secretaría de Salud; 2014. https://bit.ly/2D19dSN

9. Mann Woith W, Volchenkov G, Larson J. Russian healthcare workers' knowledge of tuberculosis and infection control. Int J Tuberc Lung Dis. 2010; 14(11): 1489-92. http://bit.ly/2zmmq7U

10. Yükseltürk N, Dinç L. Knowledge about anti-tuberculosis treatment among nurses at tuberculosis clinics. Int J Nurs Pract. 2013; 19: 47-53. https://doi.org/10.1111/ijn.12026

11. Doosti Irani A, Hashemi Shahraki A, Ghaderi E, Nasehi M, Mostafavi E. Lack of optimum practice among health care workers regarding tuberculosis in Iran: A knowledge, attitude and practice study. Am J Infect Control. 2015; 43(5): 7-12. https://doi.org/10.1016/j.ajic.2015.01.020 
12. Carvajal-Barona R, Varela-Arevalo MT, Hoyos PA, Angulo-Valencia ES, Duarte Alarcón C. Conocimientos, actitudes y prácticas frente a la tuberculosis en trabajadores del sector salud en municipios prioritarios de la costa pacífica Colombiana. Rev Cienc Salud. 2014;12(3): 339-52.

http://dx.doi.org/10.12804/revsalud12.03.2014.04

13. Batista-Sánchez MR, Alvarado-Gallegos E. Factores relacionados con la práctica del proceso de enfermería en servicios de hospitalización. Rev Enferm Inst Mex Seguro Soc. 2015; 23(2):83-90. http://bit.ly/2xkYvVs

14. Duque PA. Factores relacionados con la aplicabilidad del Proceso Atención de Enfermería en una institución de salud de alta complejidad en la ciudad de Medellín, Colombia. Rev Univ. Salud. 2014; 16(1): 91-102. http://bit.ly/2f6gzfk

15. Gutiérrez-Lesmes O. Factores que determinan la aplicación del proceso de enfermería en instituciones hospitalarias de Villavicencio, Colombia, 2008. Av. Enferm. 2009; 27(1): 60-8. http://bit.ly/2z6cro5

16. Secretaría de Salud. Dirección General y Educación en Salud. Instrucción 226/2010 SICALIDAD. México: Secretaría de Salud; 2010.

17. Arroyo-Garfias L. Trejos-Cervantes TI. Validación de un Instrumento de Valoración de Enfermería para Personas con Tuberculosis. Modelo de Virginia Henderson. [Tesis]. Ciudad de México: Universidad Nacional Autónoma de México, Escuela Nacional de Enfermería y Obstetricia; 2016.

18. González-Salvador J, Paz-Cruz G. Sistema experto para el cuidado de enfermería en Tuberculosis. [Tesis]. Ciudad de México: Universidad Nacional Autónoma de México, Facultad de Ingeniería; 2016.

19. Organización Mundial de la Salud. Temas de Salud. Enfermería. Ginebra: OMS; http://bit.ly/1DGaUf3

20. Universidad de Chile, Facultad de Medicina. La ciencia de la implementación tras las buenas políticas públicas. Chile: Universidad de Chile; 2015. https://bit.ly/2GeoiOl

21. Spiegelman D. Implementation Science and the Practice of Public Health: The Case of PEPFAR. Harvard and Tanzania Celebrate 25 Years of Collaboration; Tanzania: Africa Academy for Public Health; 2018. http://bit.ly/39itloK

22. Carrión-Robles T. La enfermería en la Sociedad Red. RIdEC. 2011; 4(1): 47-53. http://bit.ly/2yo8VHj

23. Hernández-Sampieri R, Fernández-Collado C, Baptista-Lucio P. Metodología de la investigación. $6^{a}$ ed. México: McGraw Hill; 2014. p. 126-68.

24. Secretaría de Salud. Reglamento de la Ley General de Salud en Material de Investigación para la Salud. México: DOF; 1983. http://bit.ly/1SBpqPT

25. Cruz-Martínez OA, Flórez-Suancha EL, Muñoz-Sánchez AI. Conocimientos sobre tuberculosis en trabajadores de la salud en una localidad de Bogotá D. C. Av.enferm. 2011; 23(1): 143-51. http://bit.ly/2zhNxl7

26. Van der Werf M, Langendam M, Huitric E, Manissero D. Knowledge of tuberculosis treatment prescription of health workers: a systematic review. Eur. Respir. J. 2012; 39: 1248-55. https://doi.org/10.1183/o9031936.00125611

27. Antolinez-Figueroa C, Bello-Velásquez MC, Romero-Bernal LF, Muñoz-Sánchez AI. Instrumentos y herramientas de evaluación sobre conocimientos de tuberculosis. Enfermería Glob. 2017; 48: 499-514. https://doi.org/10.6o18/eglobal.16.4.262831

28. Torres-Santiago M. Calidad de los registros clínicos de enfermería: continuidad del cuidado y seguridad del paciente. [Tesis]. Ciudad de México: Universidad Nacional Autónoma de México, Escuela Nacional de Enfermería y Obstetricia; 2011.

29. Suárez-Obando F, Gómez-Restrepo C, Castro-Díaz SM. Ciencias de la implementación: de la evidencia a la práctica. Acta med colomb. 2018; 43(4): 207-16. https://bit.ly/2YVXbDX 